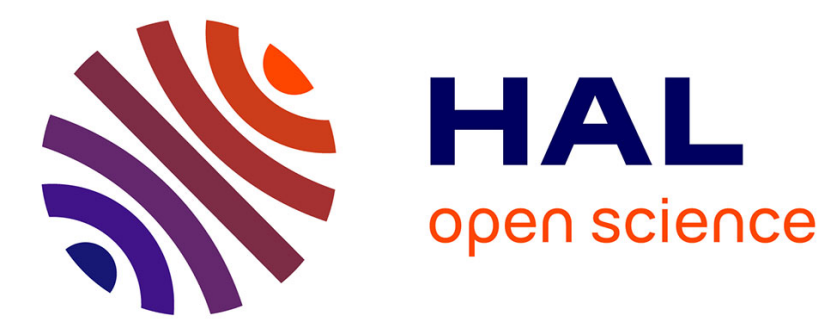

\title{
Microwave Ferrites for Cryogenic Applications
}

\author{
G. Dionne
}

\section{To cite this version:}

G. Dionne. Microwave Ferrites for Cryogenic Applications. Journal de Physique IV Proceedings, 1997, 07 (C1), pp.C1-437-C1-440. 10.1051/jp4:19971177 . jpa-00254825

\section{HAL Id: jpa-00254825 https://hal.science/jpa-00254825}

Submitted on 1 Jan 1997

HAL is a multi-disciplinary open access archive for the deposit and dissemination of scientific research documents, whether they are published or not. The documents may come from teaching and research institutions in France or abroad, or from public or private research centers.
L'archive ouverte pluridisciplinaire HAL, est destinée au dépôt et à la diffusion de documents scientifiques de niveau recherche, publiés ou non, émanant des établissements d'enseignement et de recherche français ou étrangers, des laboratoires publics ou privés. 


\title{
Microwave Ferrites for Cryogenic Applications
}

\author{
G.F. Dionne \\ Lincoln Laboratory, Massachusetts Institute of Technology, Room L-042, 244 Wood Street, Lexington, \\ MA 02173, U.S.A.
}

\begin{abstract}
Recent advances in microwave ferrite device technology have seen the introduction of superconductivity that virtually eliminates insertion losses due to electrical conduction in microstrip circuits. The conventional ferrimagnetic spinel and garnet compositions, however, are not generally optimized for temperatures in the vicinity of $77 \mathrm{~K}$ and may require chemical redesign in order to realize the full potential of these devices. For microwave transmission, absorption losses may be reduced by a natural lengthening of the spin-lattice relaxation time and the suppression of hopping electron activity at low temperatures. However, these properties could be degraded by fast-relaxing impurities that broaden ferrimagnetic resonance lines. At low temperatures, saturation magnetizations increase according to the Brillouin-Weiss function behavior that is characteristic of most magnetic materials. Increased magnetocrystalline anisotropy energies will produce greater coercive fields that will lead directly to higher hysteresis loop switching energies. Other parameters that are influenced adversely by reduced temperatures are the magnetostriction constants, which can cause significant deterioration in the stability of the remanence ratios of hysteresis loops. To modify room temperature ferrites for devices that use high- $T_{\mathrm{c}}$ superconductors, magnetochemistry must be applied to obtain the desired parameter values, which in turn must be chosen through design tradeoffs and are set by the performance requirements of particular device applications.
\end{abstract}

\section{INTRODUCTION}

In recent years the performance of microwave ferrite control devices with confined magnetic flux [1-4] has been improved dramatically through the use of low-loss superconducting circuits. Microstrip phase shifters and switching circulators employing meanderline or other circuitry that produces quasi-circular polarization have increased the importance of ferrites for use at cryogenic temperatures. Superconducting devices demonstrated to date have employed commercially available ceramic ferrites designed for room temperature operation. At liquid nitrogen temperatures, all of the relevant ferrite properties are significantly different from their room temperature values, and the values at these low-temperature operating conditions can be critical in the design for optimum device performance. In some cases, such as fixed-bias circulators used with cryogenically cooled low noise amplifiers, composition adjustments of calcium-vanadium garnet ferrites have provided low loss media for use in receivers that operate at frequencies below the millimeter-wave bands. For devices that require rapid changes of the magnetic state, however, the ferrite design is more critical. The influence of magnetocrystalline anisotropy and magnetostriction on the shape of the hysteresis loops can be crucial in determining the stability and efficiency of nonreciprocal switching devices. Except for devices like the $\mathrm{Y}$-junction circulator in which the superconductor circuit and ferrite disc combination are placed directly in the flux of an external magnetic field [5], the loop shape is a major determinant in the efficiency of switching or tunable devices that take advantage of a variable magnetization state.

In this paper the limitations of conventional ferrites for cryogenic applications will be examined and design alterations to achieve optimum performance will be proposed. Device geometries and the potential need for ferrite as single crystals or epitaxial films for improved physical compatibility with the superconductors will be described first.

\section{DEVICE CONFIGURATIONS}

A prototype structure of a microwave ferrite-superconductor (FSC) phase shifter $[1,2]$ is shown in Figure 1. In this configuration, which would apply for metallic niobium superconductor deposited directly on a ferrite substrate, the circuit is patterned in the form of a meanderline to create the effect of circular polarization necessary for the nonreciprocal properties of phase shifters and ring-network circulators [4]. To avoid penetration of the superconductor by magnetic fields that would degrade its low-loss performance, the magnetic flux inside the magnetized ferrite substrate is confined to a toroidal structure by 
a U-shaped ferrite return path. By this means the magnetic field component of the microwave signal carried by the superconductor may interact gyromagnetically with the magnetization $4 \pi M$ of the ferrite substrate without the magnetic flux degrading the performance of the superconductor. For high- $T_{c}$ oxide $\mathrm{YBa}_{2} \mathrm{Cu}_{3} \mathrm{O}_{7}(\mathrm{YBCO})$, the task of arranging the circuit in close contact with the ferrite required a more complicated structure [3] because the YBCO had to be deposited on a single crystal $\mathrm{LaAlO}_{3}$ (or $\mathrm{MgO}$ ) dielectric substrate. With the generic planar structure proposed in Figure 2 , high- $T_{\mathrm{c}}$ films deposited in a controlled proximity to ferrite substrates through the use of buffer layers [6] would provide the ferrite-superconductor interactions necessary for good microwave performance. Regardless of the particular FSC device configuration, however, the ferrite properties that have proven effective at room temperature must also be preserved for low temperature operation.

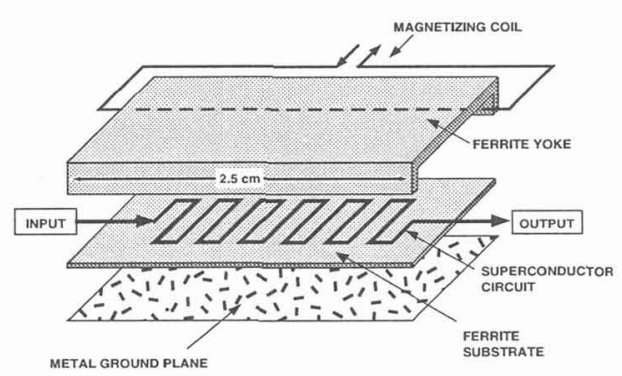

Figure 1: Prototype X-band FSC phase shifter with two-piece magnetic toroid circuit.

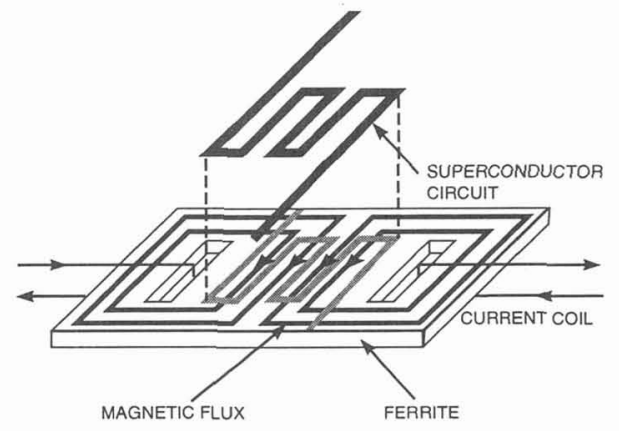

Figure 2: Planar FSC phase shifter concept.

\section{DIELECTRIC LOSS TANGENT}

For all microwave applications, a major concern is power loss through dielectric absorption mechanisms. These effects are reflected by the imaginary part of the complex permittivity $\varepsilon=\varepsilon^{\prime}-\mathrm{j} \varepsilon^{\prime \prime}$, which is usually expressed as the dielectric loss tangent $\tan \delta_{\varepsilon}=\varepsilon^{\prime \prime} / \varepsilon^{\prime}$. In ferrites where thermally activated electron hopping by the reaction $\mathrm{Fe}^{2+} \leftrightarrow \mathrm{Fe}^{3+}+\mathrm{e}^{-}$causes a conductivity $\sigma$, the dielectric loss can be expressed as the temperature dependent function

$$
\tan \delta_{\varepsilon}=\frac{\sigma}{2 \pi v \varepsilon^{\prime}}=\frac{n \mathrm{e}^{2} D}{2 \pi v \varepsilon^{\prime} \mathrm{k} T} e^{-\frac{E_{\mathrm{hop}}}{\mathrm{k} T}},
$$

where $n$ is the density of carriers, $D$ is the diffusion constant, $E_{\text {hop }}$ is the activation energy, and $v$ is the frequency of the microwave signal. Typically $E_{\text {hop }} \sim 0.1-0.5 \mathrm{eV}$, and the electron hopping activity should decrease by several orders of magnitude as $T$ is lowered from $300 \mathrm{~K}$ to $77 \mathrm{~K}$. Suppression of hopping conduction should reduce dielectric loss tangents particularly at lower frequencies, as indicated by Eq. (1).

\section{MAGNETIC LOSS TANGENT}

At room temperature, loss through gyromagnetic effects are represented by the imaginary part of the microwave complex microwave permeability $\mu=\mu^{*}-\mathrm{j} \mu^{\prime \prime}$ or magnetic loss tangent tan $\delta_{\mu}=\mu^{\prime \prime} / \mu^{\prime}$. With the exception of filters that operate at the center of the ferrimagnetic resonance (FMR) line, phase shift and Faraday rotation devices are designed for frequencies far from FMR. Ferrite materials for these applications are selected on the basis of narrow intrinsic magnetic field linewidths $\Delta H_{i}$, which influence the loss through the relation $\tan \delta_{\mu} \sim \gamma \Delta H_{\mathrm{i}} / \nu$, where $\gamma$ is the gyromagnetic constant $(=2.8 \mathrm{GHz} / \mathrm{kOe})$.

At cryogenic temperatures, however, there are two spin-lattice relaxation mechanisms that determine the resultant loss properties. In the microwave ferrites commonly employed for control devices, the magnetic energy resides in the spin system of the collective exchange-coupled $\mathrm{Fe}^{3+}$ ions. Because $\mathrm{Fe}^{3+}$ is an $S$-state ion (orbital angular momentum is zero) the spinlattice interaction occurs through higher order perturbation effects and is therefore weak. Consequently, the microwave energy remains in the spin system for increasingly longer times as $T$ decreases. The relation for the temperature dependence of the linewidth contributed by $\mathrm{Fe}^{3+}$ ions is $\Delta H_{\mathrm{i}} \sim T^{\mathrm{n}}$, where $\mathrm{n}$ is an integer that is determined by whether the interaction between spins and the lattice is through a Raman $(n=7$ or 9$)$ or the direct process $(n=1)$ [7]. It is the reduction in the density of phonons with decreasing temperature that lengthens the relaxation time and reduces the linewidth.

The second source of magnetic loss comes from fast-relaxing rare-earth $(R E)$ ions of the $4 f$ transition series that are common impurities in the starting chemical $\mathrm{Y}_{2} \mathrm{O}_{3}$ of $\mathrm{Y}_{3} \mathrm{Fe}_{5} \mathrm{O}_{12}$ (YIG). Rare-earth ions such as $\mathrm{Tb}^{3+}, \mathrm{Dy}^{3+}, \mathrm{Ho}^{3+}$, and Er ${ }^{3+}$ have strong spin-orbit coupling that leads to fast spin-lattice relaxation [8] for use in raising the critical magnetic field amplitude, given by $h_{\mathrm{c}}=\Delta H_{\mathrm{k}}(\mathrm{v} / \gamma 4 \pi M)$, where the spinwave linewidth $\Delta H_{\mathrm{k}} \approx \Delta H_{\mathrm{i}}$. Among the elements of the $3 \mathrm{~d}$ transition series, 
$\mathrm{Co}^{2+}$ ions have a ground state that is spin-orbit stabilized and can cause $\Delta H_{\mathrm{k}}$ increases comparable to those of the rare earth ions. For this reason, $\mathrm{Co}^{2+}$ is sometimes added to spinels ferrites to raise peak power thresholds. For the conventional FMR fast-relaxation case, Seiden [9] concluded that the rare-earth contribution to $\Delta H_{\mathrm{i}}$ is given by

$$
\begin{aligned}
& \Delta H_{\mathrm{i}}^{\mathrm{RE}}=C H \frac{\hbar \omega_{e x}}{\mathrm{k} T}\left(\omega_{e x} \tau\right) \quad \text { for } \omega_{e x} \tau \ll 1 \text { at high } T, \\
& \Delta H_{\mathrm{i}}^{\mathrm{RE}}=C H\left(\omega_{e x} \tau\right)^{-1} \quad \text { for } \omega_{e x} \tau \gg 1 \text { at low } T,
\end{aligned}
$$

where $C$ is the concentration of fast relaxing ions, $\hbar \omega_{e x}$ is the exchange energy which decreases to zero at the Curie temperature, and $\tau \propto T^{-n}$ is the spin-lattice relaxation time of the rare-earth ions. It is evident by inspection of Eq. (2) that $\Delta H_{1}$ reaches a maximum at a temperature between $0 \mathrm{~K}$ and the Curie temperature. In Figure 3 , theory and experiment are compared for a typical case, (Ho,Y)IG, where it is seen that $\Delta H_{\mathrm{i}}$ reaches a peak value at $T \sim 77 \mathrm{~K}$ that is more than an order of magnitude greater than the value at room temperature. For general applications where average power magnetic loss must be minimized, even impurity levels of fast-relaxing ions may have to be eliminated.

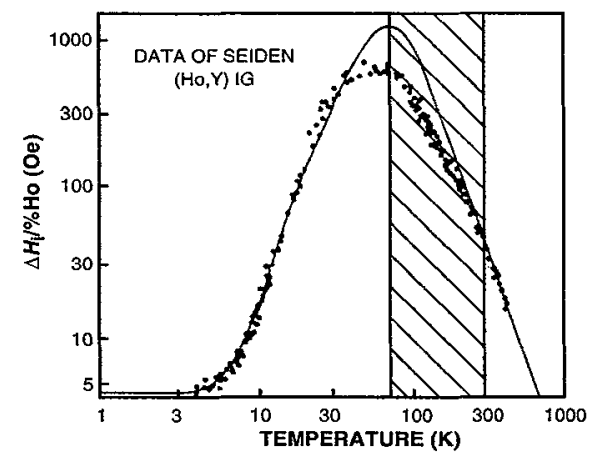

Figure 3: $\mathrm{X}$-band FMR linewidth per percentage of $\mathrm{Ho}^{3+}$ substitution in YIG as a function of temperature. Solid curve was calculated by Seiden also [9].

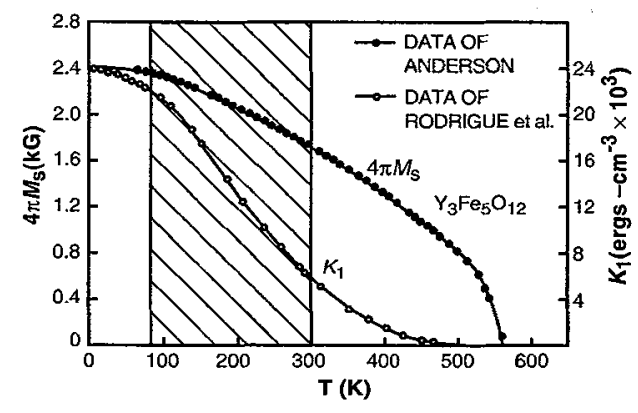

Figure 4: Variation of magnetization and magnetocrystalline anisotropy of YIG as a function of temperature.

\section{MAGNETIZATION AND HYSTERESIS LOOPS}

Microwave ferrite devices rely on the strength of the gyromagnetic interaction between the magnetic field of the signal and the magnetization of the ferrite. In most compositions, including those with modest concentrations of $\mathrm{Gd}^{3+}$ ions, $4 \pi M$ increases with reduced temperature and care must be taken to account for significant changes, as shown for YIG [10] in Figure 4. These increases in $4 \pi M$ can be beneficial for use at frequencies above $\mathrm{X}$ band, particularly in the NiZn or LiZn spinel ferrites where magnetization values greater than $7000 \mathrm{G}$ can be anticipated [11].

With the exception of devices like the Y-junction circulators that operate in the fully magnetized state with a ferrite disc arranged with its axis normal to an external magnetic field, most of the commonly used devices operate in the partially magnetized state by selection of a magnetic state from a minor hysteresis loop [12]. These devices require hysteresis loops of stable remanent magnetization and low hysteresis energy for fast and efficient switching of the magnetic state.

There are two important considerations in the choice of a ferrite for applications that involve hysteresis loops the loop squareness, characterized by the ratio of remanent magnetization to saturation magnetization (remanence ratio $R=$ $4 \pi M_{\mathrm{p}} / 4 \pi M_{\mathrm{s}}$ ), and the coercive field $H_{c}$. Both of these parameters are sensitive to temperature and are related through the magnetoelastic properties of magnetocrystalline anisotropy and magnetostriction. In Figure 5, a sample hysteresis loop of ceramic YIG measured at $60 \mathrm{~Hz}$ is presented to illustrate a nearly tenfold increase in $H_{\mathrm{c}}$. As the temperature is lowered from $300 \mathrm{~K}$ to $77 \mathrm{~K}$, the anisotropy constant $K_{1}$ that controls $H_{\mathrm{c}}$ increases by a factor of five as shown for YIG [13] in Figure 4. It may readily be inferred that the related "anisotropy field" parameter $K_{1} / M$ also increases, but not as severely (provided that $\mathrm{Gd}^{3+}$ ions are not present to offset the increase in $\left.4 \pi M\right)$. Not surprisingly, the magnitudes of the isotropic magnetostriction constant $\langle\lambda\rangle=(2 / 5) \lambda_{100}+(3 / 5) \lambda_{111}$ plotted for YIG [14] in Figure 6 also increases significantly over this temperature range. For operation at cryogenic temperatures, sensitivity of $4 \pi M$ to temperature can be less of a problem than at room temperature because of the flatness of the Brillouin function behavior in the low temperature range. Moreover, cryogenic systems are likely to operate in a controlled-temperature environment. The sensitivity of $R$ to stress, however, can be a more serious problem if $K_{1}$ is reduced by chemical design to maintain a low $H_{\mathrm{c}}$ for efficient switching. Then the stress sensitivity may increase dramatically due to larger values of $\langle\lambda\rangle$. As reported previously [15], the individual $\lambda_{100} / K_{1}$ and $\lambda_{111} / K_{1}$ ratios can determine the stress sensitivity of $R$. To counter this effect, further adjustments in the magnetoelastic properties will be necessary, but at the risk of increased magnetic loss. 


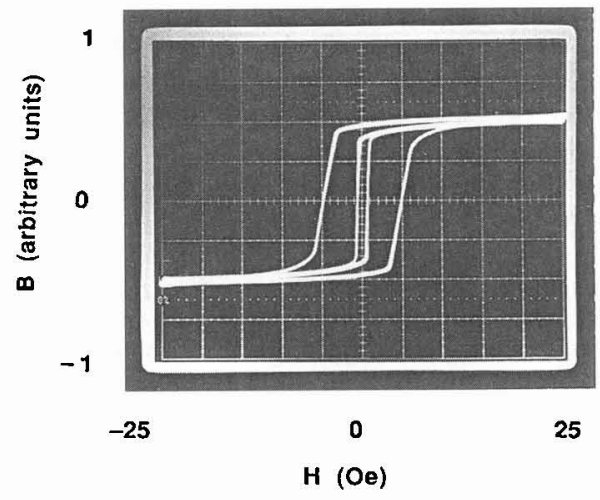

Figure 5: Hysteresis loops of ceramic YIG at $300 \mathrm{~K}$ and $77 \mathrm{~K}$.

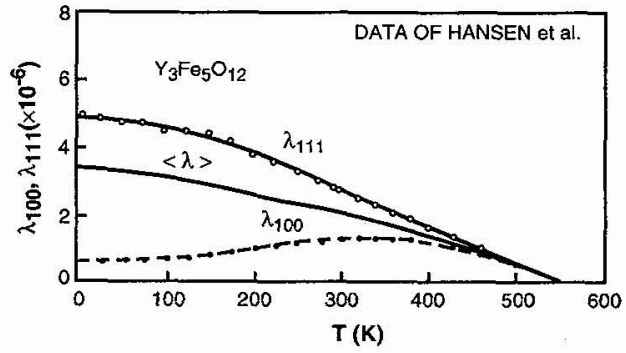

Figure 6: Magnetostriction constants of YIG as a function of temperature.

\section{CONCLUSIONS}

Redesign of the garnet and spinel ferrite compositions for optimum device performance at cryogenic temperatures may be accomplished by means of standard chemical substitutions. For this purpose two strategies are available: magnetic dilution whereby $\mathrm{Fe}^{3+}$ (or other) magnetic ions are replaced by diamagnetic substitutions such as $\mathrm{Al}^{3+}, \mathrm{Ga}^{3+}, \mathrm{In}^{3+}, \mathrm{Zn}^{2+}$ to tailor magnetization and anisotropy behavior; also $\mathrm{Co}^{2+}$ and $\mathrm{Mn}^{3+}$ or rare-earth ions may be introduced in small concentrations to tailor the magnetoelastic properties. Because of the magnetic loss properties of the rare-earth series, the use $\mathrm{Gd}^{3+}$ in high concentrations to shape the thermomagnetization curves must be avoided. Where rare-earth impurities have an adverse effect on magnetic loss in the garnets, replacement of $\mathrm{Y}^{3+}$ may be accomplished by $\mathrm{Bi}^{3+}$ substitutions [16], or by adopting a version of $2 \mathrm{Ca}^{2+}-\mathrm{V}^{5+}$ iron garnet (CVIG) system, which is already used for fixed-bias circulators with cryogenically cooled low noise amplifiers. Narrow resonance linewidths are particularly important for high- $Q$ ferrite filters.

To establish a catalog of microwave ferrites for a variety of cryogenic applications, renewed programs of fundamental measurements at low temperatures should be undertaken for the purpose of enabling the chemical design necessary to cope with the complex tradeoffs involved in device performance optimization.

\section{Acknowledgments}

The author is grateful for the support of the Analog Device Technology Group of MIT Lincoln Laboratory. This work was sponsored by the Department of the Air Force.

\section{References}

[1] Dionne G.F., Oates D.E., and Temme D.H., IEEE MTT-S Intl. Microwave Symp. Digest (1994) 101-103.

[2] Dionne G.F., Oates D.E., and Temme D.H., IEEE Trans. Magn. 30 (1994) 4518-4520.

[3] Dionne G.F., Oates D.E., and Temme D.H., IEEE Trans. Appl. Supercond. 5 (1995) 2083-2086.

[4] Weiss J.A., Dionne G.F., and Temme D.H., IEEE Trans. Microwave Th. \& Techn. 43 (1995) 2743-2748.

[5] Denlinger E.J., Paglione R., Kalokitis D., Belohoubek E., Piqué A., Wu X.D., Venkatesan T., Fathy A., Pendrick V., Green, S., and Mathews S., IEEE Microwave and Guided Wave Letts. 2 (1992) 449-451.

[6] Piqué A., Harshavardhan K.S., Moses J., Mathur M., Belohoubek E., Venkatesan T., Denlinger E.J., Kaliotis D., Fathy A., Pendrick V., Rajeswari M., and Jiang W., Appl. Phys. Lett. 67 (1995) 1778-1780.

[7] Standley K.J. and Vaughan R.A., "Electron Spin Relaxation Phenomena in Solids," (Plenum, New York, 1969) p. 5.

[8] Dionne G.F., IEEE Trans. Magn. 28 (1992) 3201-3203.

[9] Seiden P.E., Phys. Rev. 133 (1964) A728-A736.

[10] Anderson E.E., Phys. Rev. 134 (1964) A1581-A1585.

[11] Dionne G.F., J. Appl. Phys. 63 (1988) 3777-3779.

[12] Ince W.J. and Temme D.H., Adv. Microwaves 4 (1969) 1-189.

[13] Rodrigue G.P., Meyer H., and Jones R.V., J. Appl. Phys. 31 (1960) 376S-382S.

[14] Hansen P., Witter K., and Tolksdorf W., Phys. Rev. 27 (1983) 6608-6625.

[15] Dionne G.F., IEEE Trans. Magn. 8 (1972) 439-443.

[16] Vittoria C., Lubitz P., Hansen P., and Tolksdorf W., J. Appl. Phys. 57 (1985) 3699-3700. 